09

\title{
Ход световых лучей в квазипериодическом волноводе или многопроходном резонаторе с плавно меняющимися свойствами
}

\author{
() В.Ю. Матьев
}

Российский федеральный ядерный центр-Всероссийский научно-исследовательский институт экспериментальной физики,

607188 Саров, Нижегородская обл., Россия

e-mail: Matev@expd.vniief.ru

(Поступило в Редакцию 15 февраля 2017 г.)

Построен ход лучей в квазипериодической оптической системе (волноводе или резонаторе), свойства которой плавно меняются по ходу луча. Вычислена лучевая матрица для большого числа проходов луча и показано, что обычное условие устойчивости (модуль следа лучевой матрицы одного прохода меньше двух) не обеспечивает ограниченности лучевой траектории после большого числа проходов.

DOI: $10.21883 /$ JTF.2017.10.45000.2208

\section{Введение}

Рассмотрена квазипериодическая оптическая система (волновод или резонатор), оптические свойства которой плавно меняются (например, из-за разогрева). Волновод

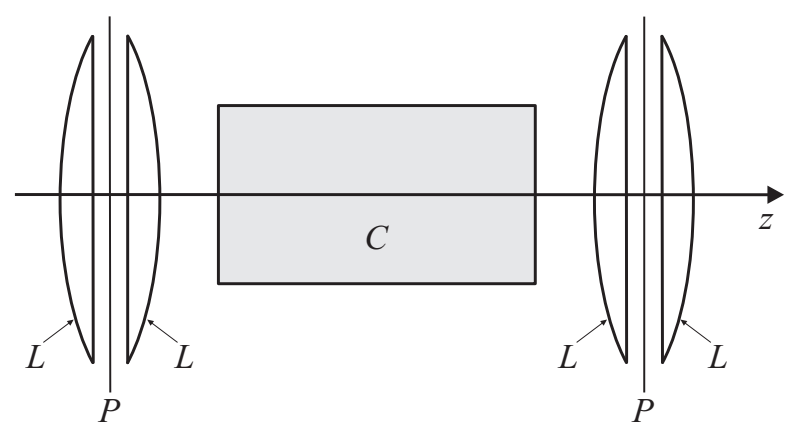

Рис. 1. Схематическое изображение периодического волновода: $P$ - опорные плоскости, разделяющие периоды волновода, $L-$ линзы, $C$ - ячейка с непрерывной средой (газовая или твердотельная), $z$ - оптическая ось.

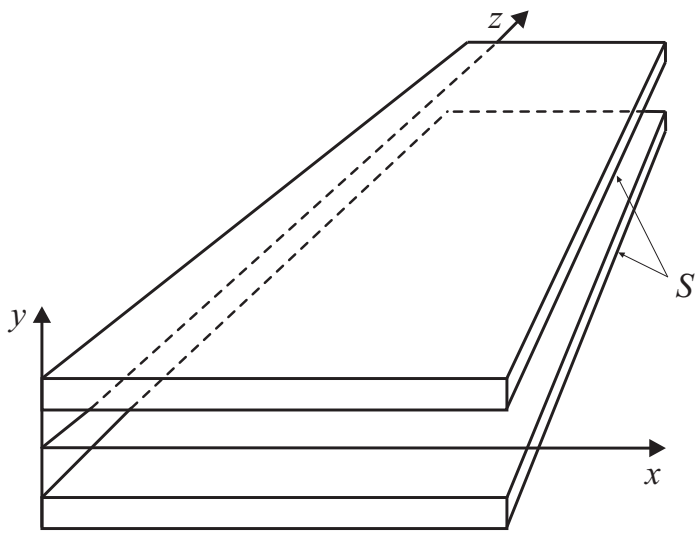

Рис. 2. Схематическое изображение прокачного лазера. $S-$ стенки кюветы, между которыми течет газ (по направлению $x$ ), $z$ - оптическая ось. может содержать как элементы, дискретно изменяющие ход лучей (линзы; резонатор - еще и зеркала), так и ячейки с непрерывным показателем преломления (с неоднородной плотностью) (рис. 1.) В рамках параксиальной оптики участок волновода (проход резонатора) характеризуется лучевой матрицей, равной произведению лучевых матриц отдельных элементов [1,2]. Плавное изменение свойств волновода означает, что соответствующие элементы лучевой матрицы для соседних участков различаются мало. Аналогом такого волновода является многопроходный лазерный резонатор с разогревом среды [3,4]. Другой пример - резонатор с прокачкой среды [5,6] (рис. 2), в котором поперечный профиль плотности развивается вдоль потока газа (по оси $x$ ). Луч при движении вдоль оптической оси $z$ отклоняется против потока газа, так что лучевая матрица одного прохода луча плавно меняется.

Итак, ход лучей через участок волновода (через резонатор) задан лучевой матрицей $\mathbf{m}_{n}$, которая немного различна для соседних участков:

$$
\left(\begin{array}{c}
y_{n} \\
\varphi_{n}
\end{array}\right)=\mathbf{m}_{n}\left(\begin{array}{c}
y_{n-1} \\
\varphi_{n-1}
\end{array}\right), \quad \mathbf{m}_{n}=\left(\begin{array}{ll}
A_{n} & B_{n} \\
C_{n} & D_{n}
\end{array}\right), \quad \varphi=\frac{d y}{d z},
$$

где $y_{n}$ - поперечное смещение луча после прохождения $n$ участков волновода ( $n$ проходов резонатора), $\varphi_{n}-$ соответствующий угол наклона лучевой траектории к оптической оси $z$. Требуется найти лучевую матрицу для $N$ участков ( $N$ проходов):

$$
\begin{gathered}
\left(\begin{array}{c}
y_{N} \\
\varphi_{N}
\end{array}\right)=\prod_{k=0}^{N-1} \mathbf{m}_{N-k}\left(\begin{array}{l}
y_{0} \\
\varphi_{0}
\end{array}\right)=\mathbf{M}_{N}\left(\begin{array}{l}
y_{0} \\
\varphi_{0}
\end{array}\right), \\
\mathbf{M}_{N}=\prod_{k=0}^{N-1} \mathbf{m}_{N-k},
\end{gathered}
$$

где $y_{0}-$ начальное смещение луча, $\varphi_{0}-$ начальный угол наклона луча. 
Плавное изменение оптических свойств по ходу луча означает, что

$$
\begin{aligned}
\left|\Delta A_{n} / A_{n}\right| \ll 1, \quad\left|\Delta B_{n} / B_{n}\right| \ll 1, \\
\left|\Delta C_{n} / C_{n}\right| \ll 1, \quad\left|\Delta D_{n} / D_{n}\right| \ll 1 .
\end{aligned}
$$

Здесь и далее $\Delta X_{n}=X_{n+1}-X_{n}$ для величины $X$; так, $\Delta A_{n}=A_{n+1}-A_{n}$, и т.д.

Матрица $\mathbf{m}_{n}$ как произведение унимодулярных лучевых матриц отдельных элементов также унимодулярна, $\operatorname{det} \mathbf{m}_{n}=A_{n} D_{n}-B_{n} C_{n}=1$.

\section{Ход лучей через многопроходный резонатор}

Матрицу полного прохода луча через резонатор можно симметризовать по главной диагонали, $A=D$. Действительно, зеркало радиуса $r$ оптически эквивалентно системе из плоского зеркала и линзы с фокусным расстоянием $f=r$ [7]; совместим опорные плоскости резонатора с плоскими зеркалами (рис. 1). Если ход лучей в резонаторе развернуть вдоль оптической оси, то получится картина, аналогичная периодическому волноводу. Для произвольной $a b c d$-матрицы w полупрохода резонатора от одной опорной плоскости до другой обратная матрица $\mathbf{w}^{-1}$, матрица обратного полупрохода $\mathbf{w}_{R}$ и матрица полного прохода $\mathbf{m}$ имеют вид [1]

$$
\begin{gathered}
\mathbf{w}=\left(\begin{array}{ll}
a & b \\
c & d
\end{array}\right), \quad \mathbf{w}^{-1}=\left(\begin{array}{cc}
d & -b \\
-c & a
\end{array}\right), \quad \mathbf{w}_{R}=\left(\begin{array}{ll}
d & b \\
c & a
\end{array}\right) ; \\
\mathbf{m}=\mathbf{w}_{R} \mathbf{w}=\left(\begin{array}{ll}
d a+b c & d b+b d \\
c a+a c & c b+a d
\end{array}\right) .
\end{gathered}
$$

Элементы матрицы m можно выразить через тригонометрические (или гиперболические) функции. Поскольку $A D-B C=1, A D=A^{2}=1+B C$. Если $B C<0$, то $A^{2}<1$; можно положить $A=D=\cos \Omega, \quad B C=$ $=\sin ^{2} \Omega$, задав (по модулю) ,фазу“ прохода $\Omega$. Полагая $B=\sin \Omega / \omega, C=-\omega \sin \Omega$, можно определить ,частоту“ прохода $\omega$ и знак фазы $\Omega$. Если $B C>0$, то $A^{2}>1$; $A=D= \pm \operatorname{ch} \Omega, B=\operatorname{sh} \Omega / \omega, C=\omega \operatorname{sh} \Omega$. Итак, если $B C \neq 0$, то

$$
\begin{gathered}
\mathbf{m}=\left(\begin{array}{cc}
\cos \Omega & (1 / \omega) \sin \Omega \\
-\omega \sin \Omega & \cos \Omega
\end{array}\right), B C<0, \\
\mathbf{m}=\left(\begin{array}{cc} 
\pm \operatorname{ch} \Omega & (1 / \omega) \operatorname{sh} \Omega \\
\omega \operatorname{sh} \Omega & \pm \operatorname{ch} \Omega
\end{array}\right), B C>0 .
\end{gathered}
$$

Такие матрицы описывают ход лучей через непрерывную среду с параболическим профилем показателя преломления $n^{*}(y)$ поперек оптической оси $z$ [2,7]; для убывающей параболы, $\omega^{2}=-(1 / y)\left(\partial n^{*} / \partial y\right)>0$, лучевое уравнение имеет вид $d^{2} y / d z^{2}+\omega^{2} y=0[2,7]$, а его решение

$$
y(z)=y_{0} \cos (\omega z)+\frac{\varphi_{0}}{\omega} \sin (\omega z)
$$

Следовательно, ход лучей через резонатор с плавным изменением свойств можно трактовать как прохождение луча через непрерывную среду с плавным изменением частоты $\omega=\omega(z)$. Лучевую траекторию в такой среде можно найти с помощью преобразований Лиувилля

$$
\begin{gathered}
\Omega(z)=\int_{0}^{z} \omega\left(z^{\prime}\right) d z^{\prime} \\
y(\Omega)=Y(\Omega) \exp \left(-\frac{1}{2} \int_{0}^{\Omega} \frac{1}{\omega} \frac{d \omega}{d \Omega^{\prime}} d \Omega^{\prime}\right) \\
=Y(\Omega) \sqrt{\frac{\omega(0)}{\omega(z)}} .
\end{gathered}
$$

Подставляя эти преобразования в лучевое уравнение, легко получить

$$
\begin{gathered}
\frac{d^{2} Y}{d \Omega^{2}}+W^{2}(\Omega) Y=0, \quad W^{2}(\Omega)=1+w(\Omega), \\
w(\Omega)=\frac{-1}{\sqrt{\omega(\Omega)}} \frac{d^{2}}{d \Omega^{2}}(\sqrt{\omega(\Omega)}) .
\end{gathered}
$$

Если на периоде осцилляции $z_{1}$, задаваемом условием $\omega z_{1}=\pi$, изменение частоты $\Delta \omega_{Z} \ll \omega$, то $(d \omega / d z) /\left(2 \omega^{2}\right) \cong \Delta \omega_{Z} /\left(2 \omega^{2} z_{1}\right) \ll 1, W(\Omega) \approx 1$, так что

$$
\begin{gathered}
Y(\Omega)=Y_{0} \cos \Omega+Y_{0}^{\prime} \sin \Omega, \\
Y^{\prime}(\Omega)=-Y_{0} \sin \Omega+Y_{0}^{\prime} \cos \Omega, \\
Y^{\prime}(\Omega)=\frac{1}{\omega(z)} \frac{d}{d z}\left(y(z) \sqrt{\frac{\omega(z)}{\omega(0)}}\right) \approx \frac{\varphi(z)}{\sqrt{\omega(z) \omega(0)}}, \\
Y_{0}=y_{0}, \quad Y_{0}^{\prime}=\frac{\varphi_{0}}{\omega(0)} .
\end{gathered}
$$

Аналогично ход лучей в резонаторе с плавным изменением частоты и фазы прохода для большого числа проходов можно записать в виде (2)

$$
\begin{gathered}
\left(\begin{array}{c}
y_{N} \\
\varphi_{N}
\end{array}\right)=\mathbf{M}_{N}\left(\begin{array}{c}
y_{0} \\
\varphi_{0}
\end{array}\right), \\
\mathbf{M}_{N}=\left(\begin{array}{cc}
\sqrt{\frac{\omega_{1}}{\omega_{N}}} \cos \Phi_{N} & \frac{1}{\sqrt{\omega_{N} \omega_{1}}} \sin \Phi_{N} \\
-\sqrt{\omega_{N} \omega_{1}} \sin \Phi_{N} & \sqrt{\frac{\omega_{N}}{\omega_{1}}} \cos \Phi_{N}
\end{array}\right), \\
\Phi_{N}=\sum_{k=1}^{N} \Omega_{k},
\end{gathered}
$$

где $\omega_{k}$ и $\Omega_{k}$ - частота и фаза $k$-прохода, фаза суммируется по проходам.

Действие матрицы (5) на вектор $\left(y_{n}, \varphi_{n}\right)$ эквивалентно повороту вектора $\left(y_{n}, \varphi_{n} / \omega[6)\right.$ на угол $\Omega$ без изменения длины. Действительно,

$$
\begin{gathered}
y_{n}=\cos \Omega y_{n-1}+\sin \Omega \frac{\varphi_{n-1}}{\omega}, \\
\frac{\varphi_{n}}{\omega}=-\sin \Omega y_{n-1}+\cos \Omega \frac{\varphi_{n-1}}{\omega} .
\end{gathered}
$$


Действие же матрицы (10) эквивалентно повороту вектора $\left(Y, Y^{\prime}\right)$ на угол $\Phi_{N}$, при этом инвариантна величина

$$
(\sqrt{\omega(z)} y(z))^{2}+(\varphi(z) / \sqrt{\omega(z)})^{2}=\text { const. }
$$

Для возрастающей параболы показателя преломления $n^{*}(y)$, когда $\omega^{2}=(1 / y)\left(\partial n^{*} / \partial y\right)>0$, матрица $\mathbf{M}_{N}$ аналогична (10); нужно лишь заменить $\cos \Phi_{n} \rightarrow \operatorname{ch} \Phi_{n}$, $\sin \Phi_{n} \rightarrow \operatorname{sh} \Phi_{n}$ и поменять знак в матричном элементе $\left(\mathbf{M}_{N}\right)_{21}$, cp. (5), (6); инвариант такой матрицы аналогичен (11), только будет разность квадратов вместо суммы. Матрица вида (10) и аналогичная ей гиперболическая матрица имеют групповой характер: произведение двух соседних матриц имеет тот же вид, что и исходная матрица:

$$
\begin{gathered}
\mathbf{M}\left(\omega_{n+1}, \omega_{n}, \Phi_{n}\right) \mathbf{M}\left(\omega_{n}, \omega_{n-1}, \Phi_{n-1}\right) \\
\quad=\mathbf{M}\left(\omega_{n+1}, \omega_{n-1}, \Phi_{n}+\Phi_{n-1}\right),
\end{gathered}
$$

это и позволяет легко перемножать матрицы для большого числа проходов.

Решение (10) получено эвристическим путем; оно наводит на мысль, что один проход через резонатор следует описывать матрицей вида (10), а не (5) и характеризовать тремя параметрами: фазой $\Omega$ и двумя частотами (входной частотой, характеризующей резонатор перед прохождением луча, и выходной, характеризующей резонатор после прохождением луча). Действительно, частота прохода $\omega$ плавно меняется, и выходная частота прохода равна входной частоте следующего прохода. В исходной постановке задачи частота прохода менялась скачком; это менее физично, а матрицы (5) не обладают групповым характером, если частоты проходов различны. Можно, однако, полагать, что частота прохода это выходящая частота, матрица (10) применима для нескольких проходов, а один проход луча описывается матрицей (5); это несоответствие при малых скачках частоты несущественно. Далее более строго рассмотрен общий случай.

\section{Разностные уравнения хода луча и их решение}

В общем случае квазипериодического волновода, когда $A_{n} \neq D_{n}$, матрица одного прохода не может быть записана в виде (5) или (6). Используем метод конечных разностей [7]. Для двух соседних участков, согласно (1),

$\left\{\begin{array}{l}y_{n+1}=A_{n+1} y_{n}+B_{n+1} \varphi_{n}, \\ \varphi_{n+1}=C_{n+1} y_{n}+D_{n+1} \varphi_{n},\end{array}, \quad\left\{\begin{array}{l}y_{n}=A_{n} y_{n-1}+B_{n} \varphi_{n-1}, \\ \varphi_{n}=C_{n} y_{n-1}+D_{n} \varphi_{n-1} .\end{array}\right.\right.$

Отсюда с учетом унимодулярности лучевой матрицы $\mathbf{m}_{n}$

$$
y_{n+1}-\sigma_{n+1} y_{n}+\gamma_{n+1} y_{n-1}=0,
$$

$$
\begin{gathered}
\sigma_{n+1}=A_{n+1}+D_{n}\left(B_{n+1} / B_{n}\right), \quad \gamma_{n+1}=B_{n+1} / B_{n}, \quad B_{n} \neq 0 ; \\
\varphi_{n+1}-\xi_{n+1} \varphi_{n}+\eta_{n+1} \varphi_{n-1}=0, \\
\xi_{n+1}=D_{n+1}+A_{n}\left(C_{n+1} / C_{n}\right) \\
\eta_{n+1}=C_{n+1} / C_{n}, \quad C_{n} \neq 0 .
\end{gathered}
$$

Для периодического волновода $\sigma_{n}=\xi_{n}=S=A+D$, $\gamma_{n}=\eta_{n}=1$. Тогда

$$
y_{n+1}-S y_{n}+y_{n-1}=0, \quad \varphi_{n+1}-S \varphi_{n}+\varphi_{n-1}=0 .
$$

Такие же уравнения были бы для резонатора (когда $A=D)$, поэтому решение для волновода можно искать в том же виде [7]

$$
y_{n}=\exp \left( \pm \Theta_{n}\right), \quad \varphi_{n}=\exp \left( \pm \Theta_{n}\right), \quad \Theta_{n}=n \theta,
$$

где $\theta-$ комплексная фаза одного участка. Подставляя (18) в (17), можно определить фазу $\theta$ (с точностью до знака) характеристическим уравнением

$$
\exp \theta+\exp (-\theta)=2 \operatorname{ch} \theta=S .
$$

Тогда общее решение уравнений (17) имеет вид

$$
\begin{gathered}
y_{n}=C_{y 1} \operatorname{ch} \Theta_{n}+C_{y 2} \operatorname{sh} \Theta_{n}, \\
\varphi_{n}=C_{\varphi 1} \operatorname{sh} \Theta_{n}+C_{\varphi 2} \operatorname{ch} \Theta_{n},
\end{gathered}
$$

cp. (7). Из начальных условий, $C_{y 1}=y_{0}$ и $C_{\varphi 2}=\varphi_{0}$, а константы $C_{y 2}$ и $C_{\varphi 1}$ определяются подстановкой в $(20)$ уравнений (12) для первого прохода

$$
\begin{aligned}
y_{1} & =y_{0} \operatorname{ch} \theta+C_{y 2} \operatorname{sh} \theta=A y_{0}+B \varphi_{0}, \\
\varphi_{1} & =C_{\varphi 1} \operatorname{sh} \theta+\varphi_{0} \operatorname{ch} \theta=C y_{0}+D \varphi_{0} .
\end{aligned}
$$

В итоге матрица $\mathbf{M}_{N}$ прохода $N$ участков принимает вид

$$
\mathbf{M}_{N}=\left(\begin{array}{cc}
\operatorname{ch} \Theta_{N}+\frac{A-\operatorname{ch} \theta}{\operatorname{sh} \theta} \operatorname{sh} \Theta_{N} & B \frac{\operatorname{sh} \Theta_{N}}{\operatorname{sh} \theta} \\
C \frac{\operatorname{sh} \Theta_{N}}{\operatorname{sh} \theta} & \operatorname{ch~} \Theta_{N}+\frac{D-\operatorname{ch} \theta}{\operatorname{sh} \theta} \operatorname{sh} \Theta_{N}
\end{array}\right),
$$

$$
\Theta_{N}=N \theta .
$$

Для ограниченной („устойчивой“) лучевой траектории $|S| \leq 2 ; \theta=i \Omega, \quad \operatorname{ch}(i \Omega)=\cos \Omega, \quad \operatorname{sh}(i \Omega)=i \sin \Omega$, где $\Omega$ - действительная фаза прохода, определяемая из (19): $2 \cos \Omega=S$; при этом матрица $\mathbf{M}_{N}$ приобретает известный вид $[1,2]$. Для неограниченной („неустойчивой““) лучевой траектории либо $S \geq 2$, и тогда $\theta=\Omega$, причем $2 \operatorname{ch} \Omega=S$; либо $S \leq-2$, и тогда $\theta=\Omega+i \pi$, $\operatorname{ch} \theta=-\operatorname{ch} \Omega, \operatorname{sh} \theta=-\operatorname{sh} \Omega$, причем $2 \operatorname{ch} \Omega=-S$.

В общем случае квазипериодического волновода удобно положить

$$
y_{n}=\sqrt{B_{n}} u_{n}, \quad \varphi_{n}=\sqrt{C_{n}} f_{n} .
$$

Тогда уравнения (13)-(16) перепишутся так:

$$
u_{n+1}-Z_{n+1} u_{n}+\Gamma_{n+1} u_{n-1}=0,
$$




$$
\begin{gathered}
f_{n+1}-\Sigma_{n+1} f_{n}+H_{n+1} f_{n-1}=0, \\
Z_{n+1}=\sigma_{n+1} \sqrt{\frac{B_{n}}{B_{n+1}}}=A_{n+1} \sqrt{\frac{B_{n}}{B_{n+1}}}+D_{n} \sqrt{\frac{B_{n+1}}{B_{n}}}, \\
\Sigma_{n+1}=\zeta_{n+1} \sqrt{\frac{C_{n}}{C_{n+1}}}=D_{n+1} \sqrt{\frac{C_{n}}{C_{n+1}}}+A_{n} \sqrt{\frac{C_{n+1}}{C_{n}}}, \\
\Gamma_{n+1}=\frac{\sqrt{B_{n+1} B_{n-1}}}{B_{n}}, \quad \mathrm{H}_{n+1}=\frac{\sqrt{C_{n+1} C_{n-1}}}{C_{n}} .
\end{gathered}
$$

Согласно условиям (3) плавного изменения $B_{n}$ и $C_{n}$,

$$
\begin{aligned}
\Gamma_{n+1} & \approx \frac{\sqrt{\left(B_{n}+\Delta B_{n}\right)\left(B_{n}-\Delta B_{n-1}\right)}}{B_{n}} \\
& \approx 1-\frac{1}{2}\left(\frac{\Delta B_{n}}{B_{n}}\right)^{2} \approx 1,
\end{aligned}
$$

с точностью до членов первого порядка малости. Аналогично, $\mathrm{H}_{n+1} \approx 1$. Тогда уравнения $(25),(26)$ принимают вид, аналогичный (17):

$$
\begin{gathered}
u_{n+1}-Z_{n+1} u_{n}+u_{n-1}=0, \\
f_{n+1}-\Sigma_{n+1} f_{n}+f_{n-1}=0 .
\end{gathered}
$$

Необходимо доопределить величины $u_{0}$ и $f_{0}$, положив

$$
\begin{aligned}
& B_{0}=B_{1}^{2} / B_{2} \approx B_{1}-\Delta B_{1}, \\
& C_{0}=C_{1}^{2} / C_{2} \approx C_{1}-\Delta C_{1},
\end{aligned}
$$

где $\Delta B_{1}=B_{2}-B_{1}, \Delta C_{1}=C_{2}-C_{1}$; это следует из уравнений $(13),(15)$ для $n=1$ и согласуется с условиями $\Gamma_{1} \approx 1, \mathrm{H}_{1} \approx 1$. Отсюда следуют начальные условия для уравнений (29), (30); согласно (24),

$$
u_{0}=y_{0} / \sqrt{B_{0}}, \quad f_{0}=\varphi_{0} / \sqrt{C_{0}} .
$$

С учетом решений (9) для резонатора и (18) для волновода решение уравнения (29) для квазипериодического волновода можно искать в виде

$$
u_{N}=G_{N} \prod_{k=1}^{N} \lambda_{k}, \quad \lambda_{n}=\exp \left( \pm \theta_{n}\right)
$$

где $\theta_{n}$ - комплексная фаза участка $n$, а $G_{n}$ - амплитуда, компенсирующая изменение фазы от участка к участку. Подставляя (33) в (29), получаем

$$
G_{n+1} \lambda_{n+1} \lambda_{n}-Z_{n+1} G_{n} \lambda_{n}+G_{n-1}=0 .
$$

Характеристическое уравнение, задающее фазу $\theta_{n}$ (с точностью до знака), можно взять в виде (19): $2 \operatorname{ch} \theta_{n}=Z_{n}$. Однако величина $Z_{n}$ задается параметрами соседних проходов согласно (27). Удобно выразить $Z_{n+1}$ через параметры одного прохода. В первом порядке малости

$$
\begin{aligned}
Z_{n+1} & =\left(A_{n}+\Delta A_{n}\right) \sqrt{\frac{B_{n}}{B_{n}+\Delta B_{n}}}+D_{n} \sqrt{\frac{B_{n}+\Delta B_{n}}{B_{n}}} \\
& \approx S_{n}+\Delta A_{n}-\left(A_{n}-D_{n}\right) \frac{\Delta B_{n}}{2 B_{n}} \\
& =S_{n}+\frac{\Delta S_{n}}{2}+\frac{A_{n}-D_{n}}{2}-\left(A_{n}-D_{n}\right) \frac{\Delta B_{n}}{2 B_{n}} \\
& =S_{n}+\frac{\Delta S_{n}}{2}+\frac{B_{n}}{2} \Delta\left(\frac{R_{n}}{B_{n}}\right),
\end{aligned}
$$

где $S_{n}=A_{n}+D_{n}, R_{n}=A_{n}-D_{n}$. Зададим характеристическое уравнение

$$
\begin{aligned}
\lambda_{n}+1 / \lambda_{n} & =\exp \theta_{n}+\exp \left(-\theta_{n}\right)=2 \operatorname{ch} \theta_{n} \\
& =S_{n}+\frac{B_{n}}{2} \Delta\left(\frac{R_{n}}{B_{n}}\right),
\end{aligned}
$$

о переходе от комплексной фазы $\theta_{n}$ к действительной фазе $\Omega_{n}$ см. выше, после (23). Тогда в первом приближении

$$
\begin{gathered}
Z_{n+1}=2 \operatorname{ch} \theta_{n}+\frac{\Delta S_{n}}{2}, \\
\Delta S_{n} \approx \Delta\left(\lambda_{n}+\frac{1}{\lambda_{n}}\right) \approx \frac{\lambda_{n}^{2}-1}{\lambda_{n}^{2}} \Delta \lambda_{n} .
\end{gathered}
$$

Аналогично для уравнения (30) с учетом (28), вводя другую фазу $\psi_{n}$ :

$$
\begin{gathered}
\Sigma_{n+1}=2 \operatorname{ch} \psi_{n}+\frac{\Delta S_{n}}{2}, \\
2 \operatorname{ch} \psi_{n}=S_{n}-\frac{C_{n}}{2} \Delta\left(\frac{R_{n}}{C_{n}}\right) .
\end{gathered}
$$

Подставляя $Z_{n+1}$ из уравнений (35), (36) в уравнение (34), получаем

$$
G_{n+1} \lambda_{n+1} \lambda_{n}-G_{n}\left(\lambda_{n}^{2}+1+\frac{\lambda_{n}^{2}-1}{2 \lambda_{n}} \Delta \lambda_{n}\right)+G_{n-1}=0 .
$$

Для малых величин $\Delta \lambda_{n}=\lambda_{n+1}-\lambda_{n}$ и $\Delta G_{n}=G_{n+1}-$ $-G_{n} \approx \Delta G_{n-1}=G_{n}-G_{n-1}$; разностное уравнение (38) в первом порядке малости можно преобразовать в дифференциальное уравнение и проинтегрировать:

$$
\begin{gathered}
(G+\Delta G)(\lambda+\Delta \lambda) \lambda-G \lambda^{2}-G \frac{\lambda^{2}-1}{2 \lambda} \Delta \lambda-\Delta G \\
\approx\left(\lambda^{2}-1\right) \Delta G+G \lambda \Delta \lambda-G \frac{\lambda^{2}-1}{2 \lambda} \Delta \lambda=0 ; \\
\frac{d G}{G(\lambda)}+\frac{\lambda d \lambda}{\lambda^{2}-1}-\frac{d \lambda}{2 \lambda}=0 ; \\
G(\lambda) \sqrt{\frac{\lambda^{2}-1}{\lambda}}=G(\lambda) \sqrt{\lambda-\frac{1}{\lambda}}=G(\lambda) \sqrt{2 \operatorname{sh} \theta}=\text { const; }
\end{gathered}
$$




$$
G_{n}^{ \pm}=G_{0}^{ \pm} \sqrt{\operatorname{sh} \theta_{0} / \operatorname{sh} \theta_{n}}
$$

знаки \pm соответствуют разным знакам $\theta_{n}$. Здесь введена фаза $\theta_{0} \approx \theta_{1}-\Delta \theta_{1}=\theta_{1}-\left(\theta_{2}-\theta_{1}\right)$; это следует из условия $G_{n+1}-G_{n} \approx G_{n}-G_{n-1}$ и общего правила, см. (31). Нулевые значения параметров волновода характеризуют его состояние до прохода излучения. Итак, общее решение уравнения (13), согласно (24) и (33), записывается в виде

$$
\begin{gathered}
y_{N}=\sqrt{B_{N}}\left(G_{N}^{+} \exp \Theta_{N}+G_{N}^{-} \exp \left(-\Theta_{N}\right)\right) ; \\
\Theta_{N}=\sum_{k=1}^{N} \theta_{k} .
\end{gathered}
$$

Подставляя (39) и вводя $\quad G_{0}^{+}=\left(G_{C}+G_{S}\right) / 2$, $G_{0}^{-}=\left(G_{C}-G_{S}\right) / 2$ получаем

$$
y_{N}=\sqrt{B_{N} \frac{\operatorname{sh} \theta_{0}}{\operatorname{sh} \theta_{N}}}\left(G_{C} \operatorname{ch} \Theta_{N}+G_{S} \operatorname{sh} \Theta_{N}\right),
$$

ср. (20). Если исходить из характеристического уравнения $2 \operatorname{ch} \theta_{n}=Z_{n}$ вместо (35), то в амплитуде $G_{N}$ и фазе $\Theta_{N}$ возникнут дополнительные добавки, которые в первом приближении взаимно сократятся, так что в итоге будет тот же результат (40).

Аналогичным образом из (30) можно получить решение уравнения (15)

$$
\begin{gathered}
\varphi_{N}=\sqrt{C_{N} \frac{\operatorname{sh} \psi_{0}}{\operatorname{sh} \psi_{N}}}\left(G_{C \varphi} \operatorname{ch} \Psi_{N}+G_{S \varphi} \operatorname{sh} \Psi_{N}\right), \\
\Psi_{N}=\sum_{k=1}^{N} \psi_{k},
\end{gathered}
$$

cp. (20); угол $\psi_{n}$ определяется согласно (37). Из начальных условий

$$
G_{C}=y_{0} / \sqrt{B_{0}}, \quad G_{C \varphi}=\varphi_{0} / \sqrt{C_{0}},
$$

$G_{S}$ и $G_{S \varphi}$ можно определить, подставив в соотношения (40), (41) уравнения (12) для первого прохода: $y_{1}=A_{1} y_{0}+B_{1} \varphi_{0}, \varphi_{1}=C_{1} y_{0}+D_{1} \varphi_{0}$. Тогда

$$
\begin{aligned}
& y_{N}=\sqrt{\frac{B_{N} \operatorname{sh} \theta_{0}}{B_{0} \operatorname{sh} \theta_{N}}}\left\{y_{0} \operatorname{ch} \Theta_{N}\right. \\
& \left.+\left[\sqrt{\frac{B_{0} \operatorname{sh} \theta_{1}}{B_{1} \operatorname{sh} \theta_{0}}}\left(A_{1} y_{0}+B_{1} \varphi_{0}\right)-y_{0} \operatorname{ch} \theta_{1}\right] \frac{\operatorname{sh} \Theta_{N}}{\operatorname{sh} \theta_{1}}\right\}, \\
& \varphi_{N}=\sqrt{\frac{C_{N} \operatorname{sh} \psi_{0}}{C_{0} \operatorname{sh} \psi_{N}}}\left\{\varphi_{0} \operatorname{ch} \Psi_{N}\right. \\
& \left.+\left[\sqrt{\frac{C_{0} \operatorname{sh} \psi_{1}}{C_{1} \operatorname{sh} \psi_{0}}}\left(C_{1} y_{0}+D_{1} \varphi_{0}\right)-\varphi_{0} \operatorname{ch} \psi_{1}\right] \frac{\operatorname{sh} \Psi_{N}}{\operatorname{sh} \psi_{1}}\right\} .
\end{aligned}
$$

Фазы $\theta_{n}$ и $\psi_{n}$ определены согласно (35), (37) с точностью до знака; это не влияет на результат, поскольку гиперболические синусы входят в (42), (43) в виде отношения. Для периодического волновода, когда $\theta_{n}=\psi_{n}=\theta, B_{n}=B, C_{n}=C$, формулы (42), (43) сводятся к лучевой матрице (23). Главное отличие от случая периодического волновода (23) - наличие амплитудных факторов. Рассмотрим сначала особые случаи для уравнений (13), (29).

\section{Особые случаи}

Решение (39) неприменимо, когда $\lambda_{n} \rightarrow 1, \theta_{n} \rightarrow 0$. В этом случае происходит вырождение лучевой траектории. Согласно (27), (36), условие вырождения: $Z_{n}=2$, $\sigma_{n}=2 \gamma_{n}^{1 / 2} \approx 2+\Delta B_{n} / B_{n}$. Характеристическое уравнение (35) при этом имеет один кратный корень: $\lambda_{n} \approx 1$, $\theta_{n} \approx 0$. Здесь пролегает граница между „устойчивыми“ и „неустойчивыми“ лучевыми траекториями. Вблизи этой границы в уравнении (34) главную роль играют факторы второго порядка малости, которыми пренебрегалось при выводе (39) из (38). Именно, при $\lambda_{n}=1$, согласно (34), с учетом (24), (33)

$$
\begin{gathered}
G_{n+1}-G_{n}=G_{n}-G_{n-1}, \quad u_{n}=G_{n}=\frac{y_{n}}{\sqrt{B_{n}}}, \\
\begin{aligned}
\frac{y_{n+1}}{\sqrt{B_{n+1}}}-\frac{y_{n}}{\sqrt{B_{n}}} & =\frac{y_{n}}{\sqrt{B_{n}}}-\frac{y_{n-1}}{\sqrt{B_{n-1}}}=\ldots \\
& =\frac{y_{1}}{\sqrt{B_{1}}}-\frac{y_{0}}{\sqrt{B_{0}}}, \quad B_{0}=\frac{B_{1}^{2}}{B_{2}} .
\end{aligned}
\end{gathered}
$$

Производя суммирование этих равенств и подставляя $y_{1}$, получаем

$$
\begin{gathered}
\frac{y_{N}}{\sqrt{B_{N}}}=\left(\frac{y_{1}}{\sqrt{B_{1}}}-\frac{y_{0}}{\sqrt{B_{0}}}\right) N+\frac{y_{0}}{\sqrt{B_{0}}}, \\
y_{1}=A_{1} y_{0}+B_{1} \varphi_{0} .
\end{gathered}
$$

Это решение получено из уравнения (29) в приближении $\Gamma_{n}=1$. Более точное условие вырождения лучевой траектории можно получить из (13)

$$
\sigma_{n+1}=\sqrt{\frac{B_{n+1}}{B_{n}}}\left(1+\frac{\sqrt{B_{n+1} B_{n-1}}}{B_{n}}\right), n \geq 2,
$$

само уравнение (13) при этом можно итеративно преобразовать к виду

$$
\begin{aligned}
\frac{y_{n+1}}{\sqrt{B_{n+1}}}-\frac{y_{n}}{\sqrt{B_{n}}} & =\frac{\sqrt{B_{n+1} B_{n-1}}}{B_{n}}\left(\frac{y_{n}}{\sqrt{B_{n}}}-\frac{y_{n-1}}{\sqrt{B_{n-1}}}\right) \\
& =\sqrt{\frac{B_{n+1}}{B_{n}}} \sqrt{\frac{B_{1}}{B_{2}}}\left(\frac{y_{2}}{\sqrt{B_{2}}}-\frac{y_{1}}{\sqrt{B_{1}}}\right) .
\end{aligned}
$$

Итерируя аналогичным образом разностное уравнение для меньших индексов и производя суммирование, 
легко получить точное решение

$$
\begin{aligned}
\frac{y_{N}}{\sqrt{B_{N}}}= & \left(\frac{y_{2}}{\sqrt{B_{2}}}-\frac{y_{1}}{\sqrt{B_{1}}}\right) \sqrt{\frac{B_{1}}{B_{2}}} \\
& \times \sum_{k=3}^{N} \sqrt{\frac{B_{k}}{B_{k-1}}}+\frac{y_{2}}{\sqrt{B_{2}}}, \quad N \geq 3 .
\end{aligned}
$$

Здесь $y_{2}$ рассчитывается, согласно (13), по заданному $\sigma_{2} ; y_{1}=A_{1} y_{0}+B_{1} \varphi_{0}$.

Еще один особый случай: $\sigma_{n}=1+\gamma_{n}=2+\Delta B_{n} / B_{n}$, при этом, согласно $(27), Z_{n} \approx 2$ в первом порядке малости по $\Delta B_{n} / B_{n}$. Уравнение (13) в этом случае также легко решается

$$
\begin{aligned}
& y_{n+1}-y_{n}=\gamma_{n+1}\left(y_{n}-y_{n-1}\right) \\
& =\left(y_{1}-y_{0}\right) \prod_{k=1}^{n} \gamma_{k+1}=\left(y_{1}-y_{0}\right)\left(B_{n+1} / B_{1}\right) .
\end{aligned}
$$

Расписывая разность $y_{n}-y_{n-1}$ и т.д., после суммирования получаем

$$
y_{N}=y_{0}+\left(y_{1}-y_{0}\right) \frac{1}{B_{1}} \sum_{k=1}^{N} B_{k}, \quad y_{1}=A_{1} y_{0}+B_{1} \varphi_{0} .
$$

Примером такого случая является волновод, состоящий из участков с однородной средой длиной $l_{k}$ и показателем преломления $n_{k}^{*}$. Для такого волновода матрицы проходов $\mathbf{m}_{k}$ имеют простой вид и легко перемножаются, приводя к тому же результату, что и (47):

$$
\begin{gathered}
\mathbf{m}_{k}=\left(\begin{array}{cc}
1 & B_{k} \\
0 & 1
\end{array}\right), \quad \mathbf{M}_{N}=\left(\begin{array}{cc}
1 & P_{N} \\
0 & 1
\end{array}\right), \\
P_{N}=\sum_{k=1}^{N} B_{k}, \quad B_{k}=\frac{l_{k}}{n_{k}^{*}} .
\end{gathered}
$$

Особые случаи легче понять, записав разностное уравнение (13) в виде

$$
\begin{gathered}
\Delta^{2} y_{n}=\left(\sigma_{n+1}-1-\gamma_{n+1}\right) y_{n}+\left(\gamma_{n+1}-1\right)\left(y_{n}-y_{n-1}\right), \\
\Delta^{2} y_{n} \equiv\left(y_{n+1}-y_{n}\right)-\left(y_{n}-y_{n-1}\right)
\end{gathered}
$$

и сопоставив ему дифференциальное уравнение

$$
\frac{d^{2} y}{d z^{2}}=\frac{\sigma-1-\gamma}{(\Delta z)^{2}} y+\frac{\gamma-1}{\Delta z} \frac{d y}{d z}, \gamma-1=\frac{\Delta z}{B} \frac{d B}{d z},
$$

где $\Delta z$ - длина участка волновода. Если $\sigma=1+\gamma$, то это уравнение решается точно последовательным интегрированием

$$
\frac{1}{B(z)} \frac{d y}{d z}=\left.\frac{1}{B_{1}} \frac{d y}{d z}\right|_{0}
$$

$$
\begin{aligned}
y & =y_{0}+\left.\frac{d y}{d z}\right|_{0} \frac{1}{B_{1}} \int_{0}^{z} B\left(z^{\prime}\right) d z^{\prime} \\
& =y_{0}+\frac{\varphi_{0}}{B_{1}} \int_{0}^{z} B\left(z^{\prime}\right) d z^{\prime},
\end{aligned}
$$

где $B_{1}=B(0)$; этот случай соответствует решению (47).

Если $\gamma \neq 1$, то в уравнении (48) следует перейти к новой переменной $u(z)$ :

$$
\begin{gathered}
y(z)=\exp \left(\frac{P(z)}{2}\right) u(z)=u(z) \sqrt{B(z)}, \\
P(z)=\int \frac{\gamma-1}{\Delta z} d z=\ln B(z) \\
\frac{d^{2} u}{d z^{2}}=\left[\frac{\sigma-1-\gamma}{(\Delta z)^{2}}+\frac{3}{4}\left(\frac{1}{B} \frac{d B}{d z}\right)^{2}-\frac{1}{2 B} \frac{d^{2} B}{d z^{2}}\right] u
\end{gathered}
$$

это соответствует переходу от (13) к (25), (29); функция $u(z)$ соответствует амплитуде $u_{n}$. В правой части (50) второе и третье слагаемые в скобках можно опустить как величины второго порядка малости; тогда

$$
\frac{d^{2} u}{d z^{2}} \approx \frac{\sigma-1-\gamma}{(\Delta z)^{2}} u
$$

Если $\sigma_{n}=2 \gamma_{n}^{1 / 2}, \quad$ то $(\sigma-\gamma-1)=-\left(\gamma_{n}^{1 / 2}-1\right)^{2} \approx$ $\approx-(\Delta z / B)^{2}(d B / d z)^{2} / 4$; правая часть (51) в первом порядке малости обращается в нуль, и тогда

$$
\begin{gathered}
\frac{d u}{d z}=\frac{d}{d z}\left(\frac{y(z)}{\sqrt{B(z)}}\right)=\text { const, } \\
\frac{y(z)}{\sqrt{B(z)}}=\frac{y_{0}}{\sqrt{B_{1}}}+\text { const } z,
\end{gathered}
$$

константа определяется по углу $\varphi_{0}$; этот случай соответствует решению (45). При $\sigma=1+\gamma$ приближенное решение (52) соответствует точному решению (49) в первом порядке малости, в чем нетрудно убедиться, положив $B(z)=B_{1}+z d B / d z$.

В окрестности $\lambda_{n}=1$, когда $\sigma_{n}=2 \gamma_{n}^{1 / 2}+\varepsilon, \varepsilon \ll 1$, (51) принимает вид

$$
\frac{d^{2} u}{d z^{2}} \approx \frac{\varepsilon(z)}{(\Delta z)^{2}} u .
$$

Если $\varepsilon(z)=\alpha z(\Delta z)^{2}$, где $\alpha-$ константа, то общее решение уравнения $u(z)$ будет линейной комбинацией функций Эйри $\mathrm{Ai}\left(\alpha^{1 / 3} z\right)$ и $\mathrm{Bi}\left(\alpha^{1 / 3} z\right)$.

Ранее предполагалось, что $B_{n} \neq 0$. Если $B_{n}=0$, то уже уравнение (13) не является корректным. В этом специфическом случае из (12) сразу имеем

$$
y_{N}=y_{0} \prod_{k=1}^{N} A_{k} \text {. }
$$

Аналогично могут быть рассмотрены особые случаи уравнений (15), (30). Далее предполагается, что $\theta_{n} \neq 0$, $B_{n} C_{n} \neq 0$. 


\section{Унификация основного решения}

Для того чтобы привести выражения (42) и (43) к матричному виду и завершить решение задачи, следует унифицировать фазы прохода $\theta_{n}$ и $\psi_{n}$, определенные соотношениями (35) и (37). Преобразуем эти соотношения

$$
\begin{aligned}
\frac{B_{n}}{2} \Delta\left(\frac{R_{n}}{B_{n}}\right) & =\frac{\sqrt{B_{n} C_{n}}}{2} \sqrt{\frac{B_{n}}{C_{n}}} \Delta\left(\frac{R_{n}}{\sqrt{B_{n} C_{n}}} \sqrt{\frac{C_{n}}{B_{n}}}\right) \\
& =2 \Delta T_{n}+R_{n} \frac{\Delta \omega_{n}}{2 \omega_{n}}, \\
\frac{C_{n}}{2} \Delta\left(\frac{R_{n}}{C_{n}}\right) & =\frac{\sqrt{B_{n} C_{n}}}{2} \sqrt{\frac{C_{n}}{B_{n}}} \Delta\left(\frac{R_{n}}{\sqrt{B_{n} C_{n}}} \sqrt{\frac{B_{n}}{C_{n}}}\right) \\
& =2 \Delta T_{n}-R_{n} \frac{\Delta \omega_{n}}{2 \omega_{n}} ; \\
\Delta T_{n}=\frac{\sqrt{\left|B_{n} C_{n}\right|}}{4} & \Delta\left(\frac{R_{n}}{\sqrt{\left|B_{n} C_{n}\right|}}\right), \quad \omega_{n}=\sqrt{\left|\frac{C_{n}}{B_{n}}\right|} .
\end{aligned}
$$

Здесь введена „частота прохода“ $\omega_{n}$. Тогда уравнения (35) и (37) для фаз прохода $\theta_{n}$ и $\psi_{n}$ можно представить в виде

$$
\begin{aligned}
& 2 \operatorname{ch} \theta_{n}=S_{n}+R_{n} \frac{\Delta \omega_{n}}{2 \omega_{n}}+2 \Delta T_{n}, \\
& 2 \operatorname{ch} \psi_{n}=S_{n}+R_{n} \frac{\Delta \omega_{n}}{2 \omega_{n}}-2 \Delta T_{n} .
\end{aligned}
$$

Введем единую комплексную фазу прохода $\vartheta_{n}$ :

$$
\begin{aligned}
2 \operatorname{ch} \vartheta_{n} & =S_{n}+R_{n} \frac{\Delta \omega_{n}}{2 \omega_{n}} \\
& =A_{n}\left(1+\frac{\Delta \omega_{n}}{2 \omega_{n}}\right)+D_{n}\left(1-\frac{\Delta \omega_{n}}{2 \omega_{n}}\right) \\
& \approx A_{n} \sqrt{\frac{\omega_{n}}{\omega_{n-1}}}+D_{n} \sqrt{\frac{\omega_{n-1}}{\omega_{n}}} ;
\end{aligned}
$$

о переходе от комплексной фазы к действительной см. после (23). Тогда

$$
\operatorname{ch} \theta_{n}=\operatorname{ch} \vartheta_{n}+\Delta T_{n}, \operatorname{ch} \psi_{n}=\operatorname{ch} \vartheta_{n}-\Delta T_{n} .
$$

Преобразуем поправку $\Delta T_{n}$ в малую добавку к фазе прохода $\delta \vartheta_{n}$ :

$$
\begin{gathered}
\theta_{n} \approx \vartheta_{n}+\delta \vartheta_{n}, \quad \psi_{n} \approx \vartheta_{n}-\delta \vartheta_{n} \\
\operatorname{ch}\left(\vartheta_{n} \pm \delta \vartheta_{n}\right) \approx \operatorname{ch} \vartheta_{n} \pm \operatorname{sh} \vartheta_{n} \delta \vartheta_{n} \\
\delta \vartheta_{n}=\Delta T_{n} / \operatorname{sh} \vartheta_{n}
\end{gathered}
$$

При расчете добавки $\delta \vartheta_{n}$ можно взять $\vartheta_{n}$ в пренебрежении поправками:

$$
\begin{gathered}
\operatorname{sh} \vartheta_{n}= \pm \sqrt{\operatorname{ch} \vartheta_{n}^{2}-1} \approx \pm \sqrt{\frac{\left(A_{n}+D_{n}\right)^{2}}{4}-1} \\
=\frac{B_{n}}{\left|B_{n}\right|} \sqrt{\frac{\left(A_{n}-D_{n}\right)^{2}}{4}+B_{n} C_{n}},
\end{gathered}
$$

знак $\vartheta_{n}$ здесь установлен для соответствия случаю (5), $(6)$, когда $A=D$. Подставляя (56) и (57) в (53), получаeM

$$
\begin{aligned}
\delta \vartheta_{n}=\frac{b \Delta L_{n}}{2 \sqrt{L_{n}^{2}+b c}}, & L_{n} & =\frac{A_{n}-D_{n}}{2 \sqrt{\left|B_{n} C_{n}\right|}}, \\
b=\frac{B_{n}}{\left|B_{n}\right|}, & c & =\frac{C_{n}}{\left|C_{n}\right|} .
\end{aligned}
$$

Малое изменение матричных элементов по ходу луча подразумевает, что $b=\mathrm{const}, c=\mathrm{const}$. Введем угол $\beta_{n}$, определенный соотношениями

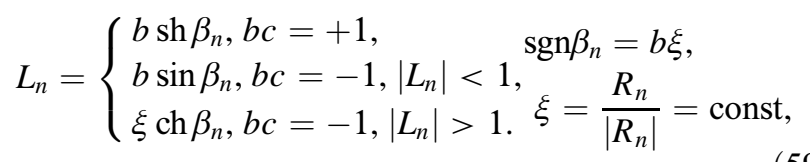

Если $b c=1$, или $b c=-1$ и $\left|L_{n}\right|>1$, то $\delta \vartheta_{n}=\Delta \beta_{n} / 2$; в этих случаях фаза прохода $\vartheta_{n}$ действительна (либо $\left.\vartheta_{n}=\Omega_{n}+i \pi\right)$ согласно (57). Если $b c=-1$ и $\left|L_{n}\right|<1$, то $\delta \vartheta_{n}=-i \Delta \beta_{n} / 2$, в этом случае фаза прохода мнимая, $\vartheta_{n}=i \Omega_{n}$.

Если в (42), (43) пренебречь в амплитудах малым различием между $B_{0}$ и $B_{1}, C_{0}$ и $C_{1}$ (эти различия не накапливаются по мере роста числа проходов), а также положить $\theta_{0} \approx \theta_{1} \approx \vartheta_{1}, \psi_{0} \approx \psi_{1} \approx \vartheta_{1}, \theta_{N} \approx \psi_{N} \approx \vartheta_{N}$, $2 \operatorname{ch} \vartheta_{1} \approx A_{1}+D_{1}$, то соотношения (42), (43) можно упростить; с учетом (55)

$$
\begin{gathered}
y_{N}=\sqrt{\frac{B_{N} \operatorname{sh} \vartheta_{1}}{B_{1} \operatorname{sh} \vartheta_{N}}}\left\{y_{0} \operatorname{ch} \Theta_{N}+\left[\frac{A_{1}-D_{1}}{2} y_{0}+B_{1} \varphi_{0}\right] \frac{\operatorname{sh} \Theta_{N}}{\operatorname{sh} \vartheta_{1}}\right\}, \\
\varphi_{N}=\sqrt{\frac{C_{N} \operatorname{sh} \vartheta_{1}}{C_{1} \operatorname{sh} \vartheta_{N}}}\left\{\varphi_{0} \operatorname{ch} \Psi_{N}+\left[\frac{D_{1}-A_{1}}{2} \varphi_{0}+C_{1} y_{0}\right] \frac{\operatorname{sh} \Psi_{N}}{\operatorname{sh} \vartheta_{1}}\right\}, \\
\Theta_{N}=\Xi_{N}+\delta \Xi_{N}, \quad \Psi_{N}=\Xi_{N}-\delta \Xi_{N}, \\
\Xi_{N}=\sum_{k=1}^{N} \vartheta_{k}, \quad \delta \Xi_{N}=\sum_{k=1}^{N} \delta \vartheta_{k} .
\end{gathered}
$$

Эти соотношения можно унифицировать, вводя приведенные переменные

$$
\begin{aligned}
\tilde{y}_{N}=\tilde{y}_{0} \operatorname{ch}\left(\Xi_{N}+\delta \Xi_{N}\right) & +\left[\frac{R_{1}}{2} \tilde{y}_{0}+b \sqrt{\left|B_{1} C_{1}\right|} \tilde{\varphi}_{0}\right] \\
& \times \frac{\operatorname{sh}\left(\Xi_{N}+\delta \Xi_{N}\right)}{\operatorname{sh} \vartheta_{1}}, \\
\tilde{\varphi}_{N}=\tilde{\varphi}_{0} \operatorname{ch}\left(\Xi_{N}-\delta \Xi_{N}\right) & -\left[\frac{R_{1}}{2} \tilde{\varphi}_{0}-c \sqrt{\left|B_{1} C_{1}\right|} \tilde{y}_{0}\right] \\
& \times \frac{\operatorname{sh}\left(\Xi_{N}-\delta \Xi_{N}\right)}{\operatorname{sh} \vartheta_{1}}, \\
\tilde{y}_{n}=y_{n} \sqrt{\left|\operatorname{sh} \vartheta_{n} / B_{n}\right|}, & \tilde{\varphi}_{n}=\varphi_{n} \sqrt{\left|\operatorname{sh} \vartheta_{n} / C_{n}\right|} .
\end{aligned}
$$

Целесообразно рассмотреть упомянутые три случая отдельно. 
1. $B_{n} C_{n}>0: \quad L_{n}=b \operatorname{sh} \beta_{n}, \quad \delta \vartheta_{n}=\Delta \beta_{n} / 2, \quad \delta \Xi_{N}=$ $=\left(\beta_{N}-\beta_{1}\right) / 2$. $\mathrm{C}$ учетом $(57)-(59)$ соотношения (62), (63) принимают вид

$$
\begin{aligned}
\tilde{y}_{N}= & \tilde{y}_{0} \operatorname{ch}\left(\Xi_{N}+\frac{\beta_{N}-\beta_{1}}{2}\right)+\left(\frac{\operatorname{sh} \beta_{1} \tilde{y}_{0}+\tilde{\varphi}_{0}}{\operatorname{ch} \beta_{1}}\right) \\
& \times \operatorname{sh}\left(\Xi_{N}+\frac{\beta_{N}-B_{1}}{2}\right), \\
\tilde{\varphi}_{N}= & \tilde{\varphi}_{0} \operatorname{ch}\left(\Xi_{N}-\frac{\beta_{N}-\beta_{1}}{2}\right)-\left(\frac{\operatorname{sh} \beta_{1} \tilde{\varphi}_{0}-\tilde{y}_{0}}{\operatorname{ch} \beta_{1}}\right) \\
& \times \operatorname{sh}\left(\Xi_{N}-\frac{\beta_{N}-B_{1}}{2}\right) .
\end{aligned}
$$

Эту систему целесообразно преобразовать к виду

$$
\begin{aligned}
\tilde{y}_{N} \operatorname{ch} \beta_{1}= & \operatorname{ch} \Xi_{N}\left(\tilde{y}_{0} \operatorname{ch} \beta_{s}+\tilde{\varphi}_{0} \operatorname{sh} \beta_{R}\right) \\
& +\operatorname{sh} \Xi_{N}\left(\tilde{y}_{0} \operatorname{sh} \beta_{S}+\tilde{\varphi}_{0} \operatorname{ch} \beta_{R}\right), \\
\tilde{\varphi}_{N} \operatorname{ch} \beta_{1}= & \operatorname{ch} \Xi_{N}\left(\tilde{\varphi}_{0} \operatorname{ch} \beta_{s}-\tilde{y}_{0} \operatorname{sh} \beta_{R}\right) \\
& -\operatorname{sh} \Xi_{N}\left(\tilde{\varphi}_{0} \operatorname{sh} \beta_{S}-\tilde{y}_{0} \operatorname{ch} \beta_{R}\right),
\end{aligned}
$$

где $\beta_{S}=\left(\beta_{N}+\beta_{1}\right) / 2, \beta_{R}=\left(\beta_{N}-\beta_{1}\right) / 2$.

Находя отсюда $\operatorname{ch} \Xi_{N}$ и $\operatorname{sh} \Xi_{N}$, можно исключить накапливаемую фазу $\Xi_{N}$ с помощью формулы $\mathrm{ch}^{2} \Xi_{N}-\mathrm{sh}^{2} \Xi_{N}=1$ и получить соотношение, связывающее конечные параметры лучевой траектории с начальными:

$\frac{\tilde{\varphi}_{N}^{2}-\tilde{y}_{N}^{2}+2 \tilde{y}_{N} \tilde{\varphi}_{N} \operatorname{sh} \beta_{N}}{\operatorname{ch}^{2} \beta_{N}}=\frac{\tilde{\varphi}_{0}^{2}-\tilde{y}_{0}^{2}+2 \tilde{y}_{0} \tilde{\varphi}_{0} \operatorname{sh} \beta_{1}}{\operatorname{ch}^{2} \beta_{1}}=$ const.

2. $B_{n} C_{n}<0, R_{n}^{2}>4\left|B_{n} C_{n}\right|: L_{n}=\xi \operatorname{ch} \beta_{n}, \delta \vartheta_{n}=\Delta \beta_{n} / 2$, $\delta \Xi_{N}=\left(\beta_{N}-\beta_{1}\right) / 2$. C учетом (57)-(59) соотношения (62), (63) принимают вид

$$
\begin{aligned}
\tilde{y}_{N}= & \tilde{y}_{0} \operatorname{ch}\left(\Xi_{N}+\frac{\beta_{N}-\beta_{1}}{2}\right)+\left(\frac{\operatorname{ch} \beta_{1} \tilde{y}_{0}+b \xi \tilde{\varphi}_{0}}{\operatorname{sh} \beta_{1}}\right) \\
& \times \operatorname{sh}\left(\Xi_{N}+\frac{\beta_{N}-\beta_{1}}{2}\right), \\
\tilde{\varphi}_{N}= & \tilde{\varphi}_{0} \operatorname{ch}\left(\Xi_{N}-\frac{\beta_{N}-\beta_{1}}{2}\right)-\left(\frac{\operatorname{ch} \beta_{1} \tilde{\varphi}_{0}+b \xi \tilde{y}_{0}}{\operatorname{sh} \beta_{1}}\right) \\
& \times \operatorname{sh}\left(\Xi_{N}-\frac{\beta_{N}-\beta_{1}}{2}\right) .
\end{aligned}
$$

Значение $\beta_{1}=0$ здесь исключается, поскольку в данном случае, согласно (57)-(59), $\beta_{1}=0$ соответствует $L_{1}^{2}=1, \vartheta_{1}=0$. Для преобразований (67), (68) также существует свой инвариант, который выводится аналогично (66):

$$
\begin{aligned}
& \frac{\tilde{\varphi}_{N}^{2}+\tilde{y}_{N}^{2}+2 b \xi \tilde{y}_{N} \tilde{\varphi}_{N} \operatorname{ch} \beta_{N}}{\operatorname{sh}^{2} \beta_{N}} \\
& =\frac{\tilde{\varphi}_{0}^{2}+\tilde{y}_{0}^{2}+2 b \xi \tilde{y}_{0} \tilde{\varphi}_{0} \operatorname{ch} \beta_{1}}{\operatorname{sh}^{2} \beta_{1}}=\text { const. }
\end{aligned}
$$

3. $B_{n} C_{n}<0, R_{n}^{2}<4\left|B_{n} C_{n}\right|$; фаза $\vartheta_{n}$ мнимая, согласно $(57) ; \vartheta_{n}=i \Omega_{n}$, где $\Omega_{n}-$ действительная фаза прохода, $\operatorname{sh}\left(\vartheta_{n}\right)=i \sin \Omega_{n}, \quad \Xi_{N}=i \Phi_{N} ; \quad \delta \vartheta_{n}=-i \Delta \beta_{n} / 2$, $\delta \Xi_{N}=-i\left(\beta_{N}-\beta_{1}\right) / 2$. Соотношения (62), (63) принимают вид

$$
\begin{aligned}
\tilde{y}_{N}= & \tilde{y}_{0} \cos \left(\Phi_{N}-\frac{\beta_{N}-\beta_{1}}{2}\right)+\left(\frac{\sin \beta_{1} \tilde{y}_{0}+\tilde{\varphi}_{0}}{\cos \beta_{1}}\right) \\
& \times \sin \left(\Phi_{N}-\frac{\beta_{N}-\beta_{1}}{2}\right), \\
\tilde{\varphi}_{N}= & \tilde{\varphi}_{0} \cos \left(\Phi_{N}+\frac{\beta_{N}-\beta_{1}}{2}\right)-\left(\frac{\sin \beta_{1} \tilde{\varphi}_{0}+\tilde{y}_{0}}{\cos \beta_{1}}\right) \\
& \times \sin \left(\Phi_{N}+\frac{\beta_{N}-\beta_{1}}{2}\right) .
\end{aligned}
$$

Эту систему легко преобразовать к виду:

$$
\begin{aligned}
\tilde{y}_{N} \cos \beta_{1}= & \cos \Phi_{N}\left(\tilde{y}_{0} \cos \beta_{s}-\tilde{\varphi}_{0} \sin \beta_{R}\right) \\
& +\sin \Phi_{N}\left(\tilde{y}_{0} \sin \beta_{S}+\tilde{\varphi}_{0} \cos \beta_{R}\right), \\
\tilde{\varphi}_{N} \cos \beta_{1}= & \cos \Phi_{N}\left(\tilde{\varphi}_{0} \cos \beta_{s}-\tilde{y}_{0} \sin \beta_{R}\right) \\
& -\sin \Phi_{N}\left(\tilde{\varphi}_{0} \sin \beta_{S}+\tilde{y}_{0} \cos \beta_{R}\right) .
\end{aligned}
$$

Находя отсюда $\cos \Phi_{N}$ и $\sin \Phi_{N}$ и складывая их квадраты, можно исключить накапливаемую фазу $\Phi_{N}$ и получить инвариант, связывающий конечные параметры лучевой траектории с начальными:

$$
\begin{aligned}
& \frac{\tilde{\varphi}_{N}^{2}+\tilde{y}_{N}^{2}+2 \tilde{y}_{N} \tilde{\varphi}_{N} \sin \beta_{N}}{\cos ^{2} \beta_{N}} \\
& =\frac{\tilde{\varphi}_{0}^{2}+\tilde{y}_{0}^{2}+2 \tilde{y}_{0} \tilde{\varphi}_{0} \sin \beta_{1}}{\cos ^{2} \beta_{1}}=Q^{2} .
\end{aligned}
$$

Формулы (70), (71) аналогичны (64), (65) с заменой гиперболических функций тригонометрическими. Однако соотношениям (70), (71) можно придать вид, аналогичный (67), (68), если положить $L_{N}=\xi \cos \beta_{n}$, $\operatorname{sgn} \beta_{n}=b \xi$ вместо (59). Стоит отметить, что фактор $\left(B_{n} / \sin \Omega_{n}\right)^{1 / 2}$ пропорционален радиусу гауссовского пучка, самовоспроизводящегося после прохождения периода с лучевой матрицей $\mathbf{m}_{n}$; фактор $\left(C_{n} / \sin \Omega_{n}\right)^{1 / 2}$ пропорционален углу расходимости такого пучка в дальней зоне [1].

Если опорные плоскости волновода выбраны так, что $A_{n}=D_{n}, R_{n}=0, \beta_{n}=0$, то выражения (70), (71) с учетом (57) принимают простой вид

$$
\begin{gathered}
\tilde{y}_{N}=\tilde{y}_{0} \cos \Phi_{N}+\tilde{\varphi}_{0} \sin \Phi_{N}, \\
\tilde{\varphi}_{N}=\tilde{\varphi}_{0} \cos \Phi_{N}-\tilde{y}_{0} \sin \Phi_{N}, \\
\tilde{y}_{n}=y_{n} \sqrt{\left|\frac{\operatorname{sh} \vartheta_{n}}{B_{n}}\right|}=y_{n} \sqrt{\omega_{n}}, \\
\tilde{\varphi}_{n}=\varphi_{n} \sqrt{\left|\frac{\operatorname{sh} \vartheta_{n}}{C_{n}}\right|}=\frac{\varphi_{n}}{\sqrt{\omega_{n}}}, \quad \omega_{n}=\sqrt{\mid \frac{C_{n}}{B_{n} \mid},}
\end{gathered}
$$




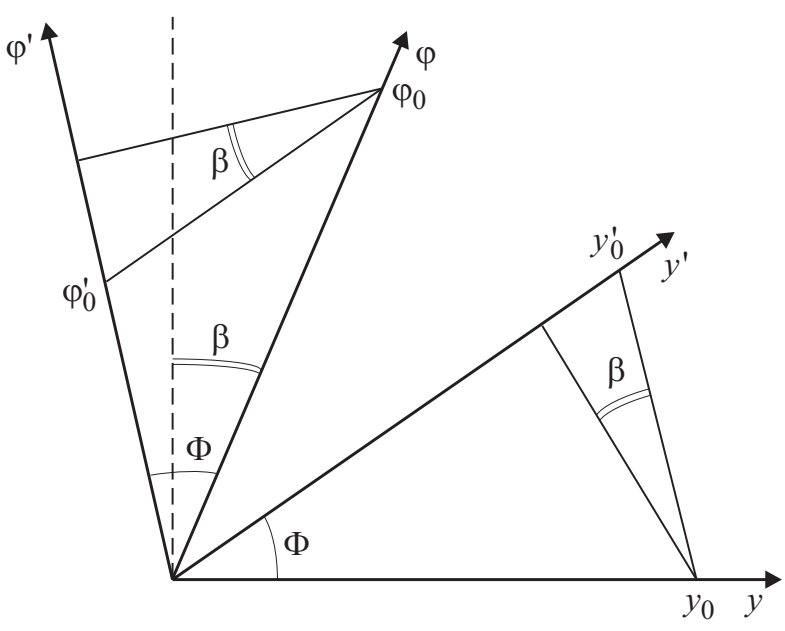

Рис. 3. Преобразование косоугольных координат при повороте системы координат на угол $\Phi$.

а инвариант (72) приобретает вид, аналогичный (11):

$\left(\sqrt{\omega_{n}} y_{n}\right)^{2}+\left(\varphi_{n} / \sqrt{\omega_{n}}\right)^{2}=$ const $=\left(\sqrt{\omega_{0}} y_{0}\right)^{2}+\left(\varphi_{0} / \sqrt{\omega_{0}}\right)^{2}$.

Таким образом, лучевая матрица (10), выведенная ранее эвристически, получила строгое обоснование. Однако исходные приближения (3) следует уточнить: изменение частоты прохода должно быть малым $\left(\Delta \omega_{n} \ll \omega_{n}\right)$ не за проход, а на периоде осцилляции, который задается условием $\Delta \Phi=\pi$.

Выражения (64), (65) при $A_{n}=D_{n}$ переходят в гиперболические формулы, аналогичные $(73)-(75)$. Выражения (67), (68) к случаю $R_{n}=0$ неприменимы.

\section{Интерпретация основного решения}

Вернемся к важнейшему случаю „устойчивой“ траектории (70), (71). Если по ходу луча $\omega=$ const, $\beta=$ const $\left(\Delta_{N} \beta=0\right)$, то лучевая матрица, выражающая вектор $\left(\tilde{y}_{N}, \tilde{\varphi}_{N}\right)$ через начальный вектор $\left(\tilde{y}_{0}, \tilde{\varphi}_{0}\right)$, имеет вид

$$
\mathbf{M}_{N}=\left(\begin{array}{cc}
\cos \Phi_{N}+\frac{\sin \beta}{\cos \beta} \sin \Phi_{N} & \frac{\sin \Phi_{N}}{\cos \beta} \\
-\frac{\sin \Phi_{N}}{\cos \beta} & \cos \Phi_{N}-\frac{\sin \beta}{\cos \beta} \sin \Phi_{N}
\end{array}\right)
$$

Эта матрица с учетом (57)-(59) соответствует матрице $(23)$ для случая $\theta=i \Omega$, но фактически матрица (77) применима в более широком смысле. Если матрица (23) по своему построению имеет групповой характер по числу проходов $n$ (фазы проходов $\theta_{n}$ одинаковы), то матрица (77) образует группу по фазе $\Phi_{n}$ : для любых фаз $\Phi_{i}$ и $\Phi_{j}$ произведение двух матриц (77) будет иметь такой же вид (77) для фазы $\Phi_{i}+\Phi_{j}$.

Геометрический смысл матрицы (77) состоит в том, что она производит поворот вектора $(\tilde{y}, \tilde{\varphi})$ на угол $\Phi$ (по часовой стрелке) в косоугольной системе координат, в которой угол между осями равен $\pi / 2-\beta$; при $\beta=0$ это будет поворот вектора $(\tilde{y}, \tilde{\varphi})$ в прямоугольной системе координат, см. (5). Действительно, при повороте системы координат $(y, \varphi)$ против часовой стрелки на угол $\Phi$ (рис. 3) косоугольная проекция отрезка $y_{0}$ на ось $y^{\prime}$ составит $y_{0}^{\prime}=y_{0}(\cos \Phi+\sin \Phi \operatorname{tg} \beta)$, а проекция отрезка $y_{0}$ на ось $\varphi^{\prime}$ будет $y_{0 \varphi}^{\prime}=-y_{0} \sin \Phi / \cos \beta$. Косоугольные же проекции отрезка $\varphi_{0}$ на оси $y^{\prime}$ и $\varphi^{\prime}$ составят $\varphi_{0 y}^{\prime}=\varphi_{0} \sin \Phi / \cos \beta$ и $\varphi_{0}^{\prime}=\varphi_{0}(\cos \Phi-\sin \Phi \operatorname{tg} \beta)$ соответственно; коэффициенты этих преобразований и составляют матрицу (77).

Если же „распрямить“ координаты и ввести новые переменные:

$$
\hat{y}=\tilde{y}+\tilde{\varphi} \sin \beta, \quad \hat{\varphi}=\tilde{\varphi} \cos \beta
$$

или

$$
\hat{y}=\tilde{y} \cos \beta, \quad \hat{\varphi}=\tilde{\varphi}+\tilde{y} \sin \beta,
$$

то они будут преобразовываться обычной матрицей поворота

$$
\left(\begin{array}{c}
\hat{y}_{N} \\
\hat{\varphi}_{N}
\end{array}\right)=\left(\begin{array}{cc}
\cos \Phi_{N} & \sin \Phi_{N} \\
-\sin \Phi_{N} & \cos \Phi_{N}
\end{array}\right)\left(\begin{array}{c}
\hat{y}_{0} \\
\hat{\varphi}_{0}
\end{array}\right) .
$$

В обоих случаях инвариантом преобразований (78) будет величина

$$
\begin{aligned}
Q_{0}^{2}=\hat{y}_{N}^{2}+\hat{\varphi}_{N}^{2} & =\tilde{y}_{N}^{2}+\tilde{\varphi}_{N}^{2}+2 \tilde{y}_{N} \tilde{\varphi}_{N} \sin \beta \\
& =\tilde{y}_{0}^{2}+\tilde{\varphi}_{0}^{2}+2 \tilde{y}_{0} \tilde{\varphi}_{0} \sin \beta
\end{aligned}
$$

равная квадрату диагонали параллелограмма, построенного на отрезках $\tilde{y}_{0}$ и $\tilde{\varphi}_{0}$ (рис. 3); инварианты (79) и (11) - это частные случаи величины (72).

В координатах $\left(\tilde{y}_{N}, \tilde{\varphi}_{N}\right)$ инвариант (72) описывает эллипс, оси которого не совпадают с координатными осями. Действительно, в координатах $(x, y)$ уравнение эллипса с полуосями $L_{x}$ и $L_{y}$, совпадающими с координатными осями (пусть для определенности $L_{y}>L_{x}$ ), имеет вид

$$
\left(\frac{x}{L_{x}}\right)^{2}+\left(\frac{y}{L_{y}}\right)^{2}=1 .
$$

При повороте координатных осей на угол $\Phi$ координаты преобразуются согласно (78); уравнение эллипса при этом приобретает вид, подобный (72):

$$
\begin{aligned}
x^{2}\left(L_{y}^{2} \cos ^{2} \Phi\right. & \left.+L_{x}^{2} \sin ^{2} \Phi\right)+y^{2}\left(L_{y}^{2} \sin ^{2} \Phi+L_{x}^{2} \cos ^{2} \Phi\right) \\
& +2 x y \sin \Phi \cos \Phi\left(L_{y}^{2}-L_{x}^{2}\right)=\left(L_{x} L_{y}\right)^{2} .
\end{aligned}
$$

В инварианте (72) коэффициенты при квадратах переменных величин $\tilde{y}$ и $\tilde{\varphi}$ совпадают; это позволяет определить из (80) угол поворота $\Phi$ :

$$
\begin{gathered}
L_{y}^{2} \cos ^{2} \Phi+L_{x}^{2} \sin ^{2} \Phi=L_{y}^{2} \sin ^{2} \Phi+L_{x}^{2} \cos ^{2} \Phi, \\
\sin ^{2} \Phi=\cos ^{2} \Phi=1 / 2,
\end{gathered}
$$

отсюда $\Phi= \pm \pi / 4$; знак угла $\Phi$ определяется знаком $\sin \beta_{N}$. Тогда из (80)

$$
x^{2}+y^{2}+\operatorname{sgn} \beta_{N} 2 x y \frac{L_{y}^{2}-L_{x}^{2}}{L_{y}^{2}+L_{x}^{2}}=2 \frac{\left(L_{x} L_{y}\right)^{2}}{L_{y}^{2}+L_{x}^{2}} .
$$


Итак, в приведенных координатах $\left(\tilde{y}_{N}, \tilde{\varphi}_{N}\right)$ инвариант (72) описывает эллипс, повернутый на угол $\pm \pi / 4$, полуоси эллипса $\left(L_{y}=L_{\max }\right.$ и $\left.L_{x}=L_{\min }\right)$ определяются соотношениями

$$
\frac{L_{\max }^{2}-L_{\min }^{2}}{L_{\max }^{2}+L_{\min }^{2}}=\sin \left|\beta_{N}\right|, 2 \frac{\left(L_{\max } L_{\min }\right)^{2}}{L_{\max }^{2}+L_{\min }^{2}}=Q^{2} \cos ^{2} \beta_{N} .
$$

В качестве наглядного примера изложенных результатов рассмотрим классический пустой резонатор, состоящий из двух зеркал с радиусами $r_{1}$ и $r_{2}$, разнесенных на расстояние $l$. Круглые зеркала можно заменить на плоские в комбинации с линзами, фокусные расстояния которых $f_{1}=r_{1}, f_{2}=r_{2}$. Совмещая опорные плоскости с плоскими зеркалами, оптическую схему резонатора можно уподобить рис. 1 (без ячейки $C$ ). Полупроход луча включает в себя преломление на линзе 1 , проход через промежуток $l$ и преломление на линзе 2 . Лучевая матрица полупрохода имеет вид

$$
\begin{aligned}
\mathbf{w} & =\left(\begin{array}{cc}
1 & 0 \\
-\frac{1}{r_{2}} & 1
\end{array}\right)\left(\begin{array}{ll}
1 & l \\
0 & 1
\end{array}\right)\left(\begin{array}{cc}
1 & 0 \\
-\frac{1}{r_{1}} & 1
\end{array}\right) \\
& =\left(\begin{array}{cc}
1-\frac{l}{r_{1}} & l \\
-\frac{1}{r_{1}}-\frac{1}{r_{2}}+\frac{l}{r_{1} r_{2}} & 1-\frac{l}{r_{2}}
\end{array}\right) .
\end{aligned}
$$

Матрица обратного полупрохода $\mathbf{w}_{R}$ имеет такой же вид, но с заменой $r_{1} \leftrightarrow r_{2}$. Перемножая $\mathbf{w}_{R} \mathbf{w}$ или просто используя (4), получаем элементы матрицы $\mathbf{m}$ полного прохода луча через резонатор

$$
\begin{gathered}
A=D=\left(1-\frac{l}{r_{1}}\right)\left(1-\frac{l}{r_{2}}\right)-\frac{l}{r_{1}}-\frac{l}{r_{2}}+\frac{l^{2}}{r_{1} r_{2}} \\
=2 g_{1} g_{2}-1 \\
B=2 l\left(1-\frac{l}{r_{2}}\right)=2 \lg _{2}, \\
C=2\left(1-\frac{l}{r_{1}}\right)\left(-\frac{1}{r_{1}}-\frac{1}{r_{2}}+\frac{l}{r_{1} r_{2}}\right)=2 \frac{g_{1}}{l}\left(g_{1} g_{2}-1\right), \\
\omega=\sqrt{\left|\frac{C}{B}\right|, \quad \frac{C}{B}=-\frac{g_{1}}{g_{2}} \frac{1-g_{1} g_{2}}{l^{2}},}
\end{gathered}
$$

где $g_{1}=1-l / r_{1}$ и $g_{2}=1-l / r_{2}-$ приведенные параметры резонатора. При таком выборе опорных плоскостей (когда обратный проход резонатора симметричен прямому проходу) $A=D$ и $\beta=0$. Условие устойчивости резонатора $|A+D|<2$ означает [2,7], что

$$
-2<4 g_{1} g_{2}-2<2 \text { или } 0<g_{1} g_{2}<1,
$$

при этом $C / B<0$. Предположим, что условие устойчивости выполняется, но резонатор плавно меняется так, что $r_{1} \rightarrow l, g_{1} \rightarrow 0$, причем это изменение за один проход невелико. В этом случае ход лучей для большого числа проходов дается выражениями (73)-(75); более наглядное представление дает инвариант (76). Если $g_{1} \rightarrow 0$, то $\omega \rightarrow 0$; при этом $y_{n} \rightarrow \infty, \varphi_{n} \rightarrow 0$. Хотя резонатор все еще остается в области устойчивости, однако световые лучи (в опорной плоскости 1) уже выходят за пределы резонатора. И наоборот: если $g_{2} \rightarrow 0$, то $\omega \rightarrow \infty$; при этом $y_{n} \rightarrow 0, \varphi_{n} \rightarrow \infty$.

Условие устойчивости (82) глобально и не зависит от выбора опорных плоскостей; в периодическом волноводе след матрицы $A+D$ инвариантен относительно сдвига опорных плоскостей (поскольку для любых двух матриц сумма диагональных элементов матрицы-произведения не зависит от порядка сомножителей). Частота прохода $\omega$, напротив, привязана к опорной плоскости, от которой строится ход лучей; если в рассмотренном выше случае проход отсчитывать от опорной плоскости 2, то $g_{1} \leftrightarrow g_{2}$. Все полученные здесь формулы для многократных проходов относятся к заданной опорной плоскости; чтобы получить координату и угол наклона луча в любой другой плоскости, нужно домножить вектор $\left(y_{N}, \varphi_{N}\right)$ на лучевую матрицу прохода от опорной плоскости до нужной.

\section{Заключение}

Получены формулы хода лучей и построены лучевые инварианты для многократных проходов волновода (резонатора) с плавным изменением свойств. Дополнительного рассмотрения требует ход лучей при переходе волноводом границы устойчивости; следует также учесть влияние на ход лучей неоднородностей коэффициента усиления.

\section{Список литературы}

[1] Джеррард А., Бёри Джс.М. Введение в матричную оптику. М.: Мир, 1978. 343 с.

[2] Ярив А. Квантовая электроника. М.: Советское радио, 1980. $488 \mathrm{c}$.

[3] Боровков В.В., Лажинцев Б.В., Мельников С.П., Мочкаев И.Н., Нор-Аревян В.А., Синянский А.А., Федоров Г.И. // Изв. АН СССР. Сер. физ. 1990. Т. 54. Вып. 10. С. 2009-2015.

[4] Матьев В.Ю., Боровков В.В., Мельников С.П. // ЖТФ. 2001. Т. 71. Вып. 1. С. 79-85.

[5] Боровков В.В., Лажсинщев Б.В., Нор-Аревян В.А., Сизов А.Н., Синянский А.А., Федоров Г.И. // Квант. электрон. 1995. Т. 22. Вып. 12. С. $1187-1191$.

[6] Матьев В.Ю. // Квант. электрон. 2003. Т. 33. Вып. 6. С. $493-$ 497.

[7] Маркузе Д. Оптические волноводы. М.: Мир, 1974. 576 с. 